\title{
GTNP BReEding BIRd Monitoring ProJeCt
}

\author{
M. L. CODY $\uparrow$ DEPARTMENT OF BIOLOGY \\ UNIVERSITY OF CALIFORNIA $\uparrow$ LOS ANGELES
}

\section{OVERVIEW: GTNP BREEDING BIRD MONITORING PROJECT}

1. Following initial independent work by M. L. Cody and $3 y$ funding from NPS, we instigated a scheme for long-term monitoring of breeding land bird populations in a wide variety of habitats representative of the northern Rockies and the Greater Yellowstone Ecosystem (GYE). Census sites are located almost entirely within Grand Teton National Park, where a broad range of representative vegetation types is accessible within close geographic proximity.

230 monitoring sites are established within and adjacent to the park in pristine habitat. Sites range from the Jackson Hole lowlands to subalpine and alpine sites, from meadow, sagebrush and marshland, through willow scrub, cottonwood and aspen woodlands, to lodgepole pine and spruce-fir forests. Some sites have a monitoring history of $>30 \mathrm{y}$; others were established in the mid-1990's.

3. The location and accessibility of the study sites permits all to be regularly and repeatedly censused during the short (6-week) breeding season. Census sites are standardized in area (5-10 ha in size) and mapped in detail (topographic features, vegetation). Census schedules, timing, and methodological protocols are established, and allow for controlled inter-site and inter-year comparisons in breeding bird populations, species composition, and densities.

\section{SEASON: COVERAGE AND PARTICIPANTS}

Participants. A selection of the 30 monitoring sites was censused by MLC (UCLA) and by Sue Wolff and associates (GTNP). Contact information is given here for the two primary participants:

Martin L. Cody

Dept. Ecology \& Evolution

University of California

Los Angeles, CA 90095-1606

PH: 310-825-1327; FX: 310-206-3987; EM: mlcody@ucla.edu

Sue Wolff

Grand Teton National Park-Resource Management

PO Box 91

Moose, WY 83102

307-739-3464; EM: susan_wolff@nps.gov

\section{Site Coverage.}

Twenty of the 30 monitoring sites were assessed in 2002. Coverage was continued on sites with the longest monitoring history, which in some cases covers several decades. While coverage spanned a wide range of habitats within the set of monitoring sites (see table 1), high elevation sites were not visited in 2002. The last column of the table indicates those sites monitored in the 2002 season; the selection continues an unbroken continuity of monitoring at important "flagship" sites, and also extends coverage of some less routinely censused areas. Overall, most of the 30 sites have been censused for about a decade. 
The Y2002 Censuses.

The list of breeding species recorded at the monitoring sites (Appendix A) now stands at 153, with the addition in 2002 of Eastern Kingbird. Y2002 was only slightly drier than average, with 573 $\mathrm{mm}$ of precipitation during the previous 12 months (August 2001-July 2002), compared to the long-term average of $606 \mathrm{~mm}$. The spring temperatures (APRJUN) were colder than normal, with monthly mean minima around $0.5 \mathrm{SD}$ below the mean, and monthly mean maxima about normal.

Breeding densities of the more common emberizid species ("sparrows") are summarized in figures $1,2$.

Figure 1

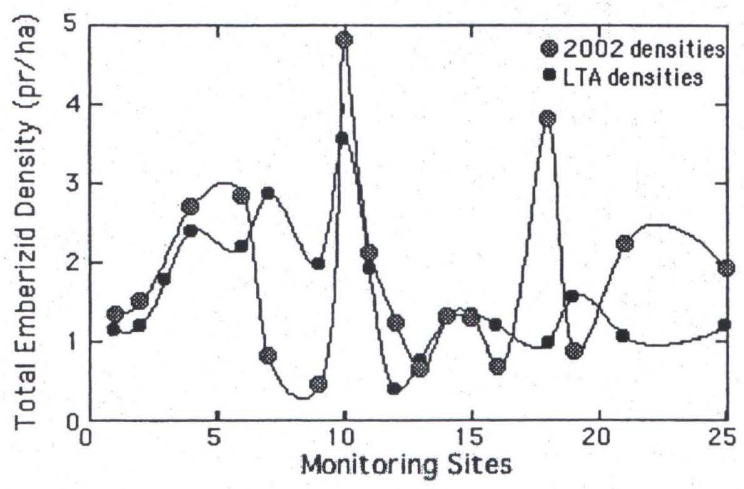

Comb ined densities of 9 emberizid species over monitoring sites, 2002 data compared to long-term average (LTA) data.

Total density for 9 emberizid species, averaged for each site over the complete data set is represented in the first figure. Density averages over $2 \mathrm{pr} / \mathrm{ha}$ from sagebrush through wet shrubby meadows to willows (sites 4-11), is lowest in the aspens (sites 12-14), and varies from 1-1.5 pr/ha in other habitats (grasslands to the left, conifer forest to the right). Data for each of the nine species summed in the first figure are shown separately.

In the second figure, the 2002 census data are shown juxtaposed against each species' average density per site, across all sites. Note that the emberizid species are distributed sequentially across habitats, from Savannah Sparrow in grasslands through Dark-eyed Junco and Chipping Sparrow in the conifer forests. In 2002, Brewer's Sparrow was particularly common in the Airport Sage (Site 6), and Lincoln's Sparrow particularly common in the Wet Willows (Site 10). Both Chipping Sparrow and Dark-eyed Junco were recorded at above average density in most of the forest sites. The potential for relating density variations in the breeding birds to on- site weather and habitat conditions, and off-site migration and overwintering conditions, increases with every additional year of the monitoring program.

Figure 2

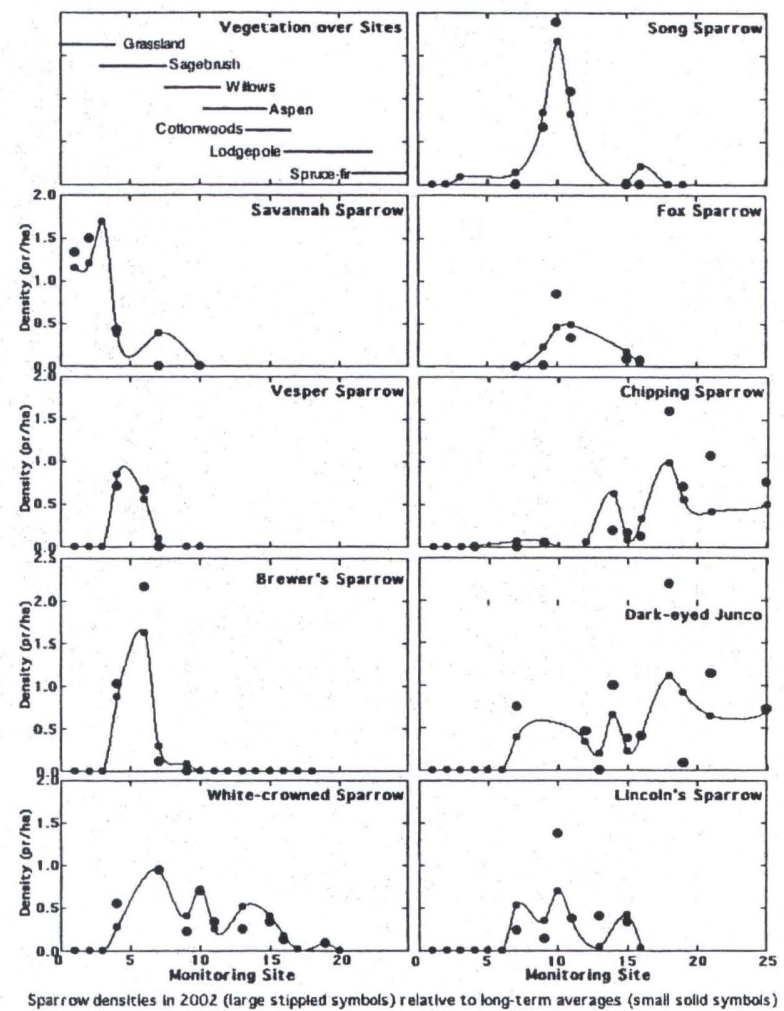


Table 1

\begin{tabular}{|c|c|c|c|c|c|c|c|c|c|c|c|c|c|c|}
\hline \multicolumn{15}{|c|}{ COVERAGE OF GTNP BREEDING BIRD MONITORING SITES, 1966-200 } \\
\hline & & & & & & & & & & & & & & Tot \\
\hline Site/Censused & 66 & 91 & 92 & 93 & 94 & 95 & 96 & 97 & 98 & 99 & 0 & 1 & 2 & Yrs \\
\hline 1:Wolf Rch & & & & 1 & 1 & 1 & 1 & 1 & & & 1 & & 1 & 7 \\
\hline 2:JLJ Sedge & & & 1 & 1 & 1 & 1 & 1 & 1 & 1 & 1 & 1 & 1 & 1 & 11 \\
\hline 3:Elk Refuge & & & & & 1 & 1 & 1 & 1 & 1 & 1 & 1 & & & 7 \\
\hline 4:JLJ Gr-sage & 1 & 1 & 1 & 1 & 1 & 1 & 1 & 1 & 1 & 1 & 1 & 1 & 1 & 13 \\
\hline 5: Ant.Flats & & & & & & 1 & 1 & 1 & & 1 & 1 & & 1 & 6 \\
\hline 6: Airpt Sage & & & & 1 & 1 & 1 & 1 & 1 & 1 & & 1 & 1 & 1 & 9 \\
\hline \multicolumn{2}{|l|}{ 7: 2OL Meadow } & & & 1 & 1 & 1 & 1 & 1 & 1 & 1 & 1 & 1 & 1 & 10 \\
\hline 8:Triangle $X$ & & & & & & & 1 & 1 & & & 1 & & & 3 \\
\hline 9: RKO & & & & 1 & 1 & 1 & 1 & 1 & 1 & 1 & 1 & 1 & 1 & 10 \\
\hline 10:JLJ Will & 1 & 1 & 1 & 1 & 1 & 1 & 1 & 1 & 1 & 1 & 1 & 1 & 1 & 13 \\
\hline 11: Oxbow & & & & 1 & 1 & 1 & 1 & 1 & 1 & 1 & 1 & 1 & 1 & 10 \\
\hline 12: Elk Rch W & 1 & & 1 & 1 & 1 & 1 & 1 & 1 & & & & & 1 & 8 \\
\hline 13: Elk Rch E & 1 & & 1 & 1 & 1 & 1 & 1 & 1 & 1 & & 1 & & 1 & 10 \\
\hline 14: Cow Lk & 1 & & 1 & 1 & 1 & 1 & 1 & 1 & & 1 & & 1 & 1 & 10 \\
\hline 15: Spread Ck & & & 1 & 1 & 1 & 1 & 1 & 1 & 1 & & 1 & & 1 & 9 \\
\hline 16: Schwabch & & & 1 & 1 & 1 & 1 & 1 & 1 & & 1 & & 1 & 1 & 9 \\
\hline 17: L. Granite & & & & & & 1 & 1 & 1 & & & 1 & & & 4 \\
\hline 18: Timbered Is & & & & & 1 & 1 & 1 & 1 & 1 & 1 & & 1 & 1 & 8 \\
\hline 19: AMK & & & 1 & 1 & 1 & 1 & 1 & 1 & 1 & 1 & 1 & 1 & 1 & 11 \\
\hline 20: TaggartLk & & & & & 1 & 1 & 1 & 1 & 1 & & & 1 & & 6 \\
\hline 21: Signal Mt & & & 1 & 1 & 1 & 1 & 1 & 1 & 1 & 1 & 1 & & 1 & 10 \\
\hline 22: Spaulding & & & & & & 1 & 1 & 1 & & & & 1 & 1 & 5 \\
\hline 23: LizardCk & & & 1 & 1 & 1 & 1 & 1 & 1 & & & & 1 & & 7 \\
\hline 24: Bradley & & & & & 1 & 1 & 1 & 1 & & & & 1 & 1 & 6 \\
\hline 25:JennyLk & & & & 1 & 1 & 1 & 1 & 1 & 1 & 1 & 1 & 1 & 1 & 10 \\
\hline 26:GranSpruce & & & & & 1 & 1 & 1 & 1 & & 1 & & & & 5 \\
\hline 27:Rendezvous & & & & & 1 & 1 & 1 & 1. & & 1 & & & & 5 \\
\hline 28:CodyBowl & & & & & 1 & 1 & 1 & 1 & & 1 & 1 & 1 & & 7 \\
\hline 29:HeronLk & & & & & & 1 & 1 & 1 & & 1 & 1 & & & 5 \\
\hline \multirow[t]{3}{*}{ 30:Blacktail } & & & & & & & 1 & 1 & & 1 & 1 & 1 & & 5 \\
\hline & & & & & & & & & & & & & & \\
\hline & & & & & & & & & & & & & & AVE \\
\hline Total Sites: & 5 & 2 & 11 & 17 & 24 & 28 & 30 & 30 & 15 & 19 & 20 & 18 & 20 & 8.22 \\
\hline
\end{tabular}




\section{APPENDIX A}

\begin{tabular}{|c|c|c|}
\hline GTNP & MONITORING SITES: & SPECIES LIST 1995-2001 \\
\hline PELECANIDAE & White Pelican & Pelecanus erythrorhynchus \\
\hline ARDEIDAE & Great Blue Heron & Ardea herodias \\
\hline GRUIDAE & Sandhill Crane & Grus canadensis \\
\hline ANATIDAE & $\begin{array}{l}\text { Trumpeter Swan } \\
\text { Canada Goose } \\
\text { Mallard } \\
\text { Gadwall } \\
\text { American Wigeon } \\
\text { Cinnamon Teal } \\
\text { Green-winged Teal } \\
\text { Blue-winged Teal } \\
\text { Redhead } \\
\text { Ring-necked Duck } \\
\text { Lesser Scaup } \\
\text { Common Merganser } \\
\text { Barrow's Goldeneye } \\
\text { Common Goldeneye } \\
\text { Bufflehead } \\
\text { Wood Duck }\end{array}$ & $\begin{array}{l}\text { Cygnus buccinator } \\
\text { Branta canadensis } \\
\text { Anas platyrhynchos } \\
\text { Anas strepera } \\
\text { Anas americana } \\
\text { Anas cyanoptera } \\
\text { Anas crecca } \\
\text { Anas discors } \\
\text { Aythya americana } \\
\text { Aythya collaris } \\
\text { Aythya affinis } \\
\text { Mergus serrator } \\
\text { Bucephala islandica } \\
\text { Bucephala clangula } \\
\text { Bucephala albeola } \\
\text { Aix sponsa }\end{array}$ \\
\hline RALLIDAE & $\begin{array}{l}\text { Virginia Rail } \\
\text { Sora } \\
\text { American Coot }\end{array}$ & $\begin{array}{l}\text { Rallus limicola } \\
\text { Porzana carolina } \\
\text { Fulica americana }\end{array}$ \\
\hline CHARADRIIDAE & $\begin{array}{l}\text { Kildeer } \\
\text { Willet }\end{array}$ & $\begin{array}{l}\text { Charadrius vociferus } \\
\text { Catoptrophorus semipalmatus }\end{array}$ \\
\hline SCOLOPACIDAE & $\begin{array}{l}\text { Long-billed Curlew } \\
\text { Spotted Sandpiper } \\
\text { Wilson's phalarope } \\
\text { Common Snipe }\end{array}$ & $\begin{array}{l}\text { Numenius americanus } \\
\text { Actitis macularia } \\
\text { Phlaropus tricolor } \\
\text { Gallinago gallinago }\end{array}$ \\
\hline LARIDAE & $\begin{array}{l}\text { Franklin's Gull } \\
\text { California Gull } \\
\text { Ring-billed gull }\end{array}$ & $\begin{array}{l}\text { Larus pipixcan } \\
\text { Larus californicus } \\
\text { Larus delawarensis }\end{array}$ \\
\hline CATHARTIDAE & Turkey Vulture & Cathartes aura \\
\hline ACCIPITRIDAE & $\begin{array}{l}\text { Golden Eagle } \\
\text { Bald Eagle }\end{array}$ & $\begin{array}{l}\text { Aquila chrysaetos } \\
\text { Haliaeetus leucocephalus }\end{array}$ \\
\hline
\end{tabular}




\begin{tabular}{|c|c|c|}
\hline & $\begin{array}{l}\text { Northern Harrier } \\
\text { Red-tailed Hawk } \\
\text { Swainson's Hawk } \\
\text { Osprey } \\
\text { Sharp-shinned Hawk } \\
\text { Cooper's Hawk } \\
\text { Northern Goshawk }\end{array}$ & $\begin{array}{l}\text { Circus cyaneus } \\
\text { Buteo jamaicensis } \\
\text { Buteo swainsoni } \\
\text { Pandion haliaetus } \\
\text { Accipiter striatus } \\
\text { Accipiter cooperi } \\
\text { Accipiter gentilis }\end{array}$ \\
\hline FALCONIDAE & $\begin{array}{l}\text { American Kestrel } \\
\text { Prairie Falcon } \\
\text { Peregrine Falcon }\end{array}$ & $\begin{array}{l}\text { Falco tinnunculus } \\
\text { Falco mexicanus } \\
\text { Falco peregrinus }\end{array}$ \\
\hline PHASIANIDAE & $\begin{array}{l}\text { Ruffed Grouse } \\
\text { Blue Grouse } \\
\text { Sage Grouse }\end{array}$ & $\begin{array}{l}\text { Bonasa umbellus } \\
\text { Dendrogapus obscurus } \\
\text { Centrocercus urophasianus }\end{array}$ \\
\hline COLUMBIDAE & Mourning Dove & Zeneadura macroura \\
\hline STRIGIDAE & $\begin{array}{l}\text { Great Gray Owl } \\
\text { Great Horned Owl } \\
\text { Long-eared Owl } \\
\text { Short-eared owl } \\
\text { Northern Pygmy-Owl }\end{array}$ & $\begin{array}{l}\text { Strix nebulosa } \\
\text { Bubo virginianus } \\
\text { Asio otus } \\
\text { Asio flammeus } \\
\text { Glaucidium gnoma }\end{array}$ \\
\hline CAPRIMULGIDAE & Common nighthawk & Phalaenoptilus nuttalli \\
\hline TROCHILIDAE & $\begin{array}{l}\text { Calliope Hummingbird } \\
\text { Broad-tailed hummingbird } \\
\text { Rufous hummingbird }\end{array}$ & $\begin{array}{l}\text { Stellula caliope } \\
\text { Selasphorus platycercus } \\
\text { Selasphorus rufus }\end{array}$ \\
\hline ALCENIDAE & Belted Kingfisher & Ceryle alcyon \\
\hline PICIDAE & $\begin{array}{l}\text { Northern Flicker } \\
\text { Williamson's Sapsucker } \\
\text { Red-naped Sapsucker } \\
\text { Downy Woodpecker } \\
\text { Hairy Woodpecker } \\
\text { Three-toed Woodpecker } \\
\text { Black-backed Woodpecker }\end{array}$ & $\begin{array}{l}\text { Colaptes auratus } \\
\text { Sphyrapicus thyroides } \\
\text { Sphyrapicus nuchalis } \\
\text { Picoides pubescens } \\
\text { Picoides villosus } \\
\text { Picoides tridactylus } \\
\text { Picoides arcticus }\end{array}$ \\
\hline TYRANNIDAE & $\begin{array}{l}\text { Western Wood Pewee } \\
\text { Olive-sided Flycatcher } \\
\text { Say's Phoebe } \\
\text { Dusky Flycatcher } \\
\text { Hammond's Flycatcher } \\
\text { Least Flycatcher }\end{array}$ & $\begin{array}{l}\text { Contopus sordidulus } \\
\text { Nuttallornis borealis } \\
\text { Sayornis saya } \\
\text { Empidonax oberholseri } \\
\text { Empidonax hammondii } \\
\text { Empidonax minimus }\end{array}$ \\
\hline
\end{tabular}




\begin{tabular}{|c|c|c|}
\hline & $\begin{array}{l}\text { Willow flycatcher } \\
\text { Cordilleran Flycatcher } \\
\text { Eastern kingbird }\end{array}$ & $\begin{array}{l}\text { Empidonax traillii } \\
\text { Empidonax occidentalis } \\
\text { Tyrannus tyrannus }\end{array}$ \\
\hline HIRUNDINIDAE & $\begin{array}{l}\text { Tree Swallow } \\
\text { Violet-green swallow } \\
\text { Rough-winged Swallow } \\
\text { Cliff Swallow } \\
\text { Barn Swallow } \\
\text { Bank Swallow }\end{array}$ & $\begin{array}{l}\text { Tachycineta bicolor } \\
\text { Tachycineta thalassina } \\
\text { Stelgidopteryx serripennis } \\
\text { Hirundo pyrrhonota } \\
\text { Hirundo rustica } \\
\text { Riparia riparia }\end{array}$ \\
\hline CORVIDAE & $\begin{array}{l}\text { Steller's Jay } \\
\text { Gray Jay } \\
\text { Clark's Nutcracker } \\
\text { Black-billed Magpie } \\
\text { American Crow } \\
\text { Common Raven }\end{array}$ & $\begin{array}{l}\text { Cyanositta stelleri } \\
\text { Perisoreus canadensis } \\
\text { Nucifraga columniana } \\
\text { Pica pica } \\
\text { Corvus brachyrhynchos } \\
\text { Corvus corax }\end{array}$ \\
\hline PARIDAE & $\begin{array}{l}\text { Black- capped Chickadee } \\
\text { Mountain Chickadee }\end{array}$ & $\begin{array}{l}\text { Parus atricapillus } \\
\text { Parus gambeli }\end{array}$ \\
\hline CERTHIDAE & Brown Creeper & Certhia americana \\
\hline SITTIDAE & $\begin{array}{l}\text { White-breasted Nuthatch } \\
\text { Red-breasted Nuthatch }\end{array}$ & $\begin{array}{l}\text { Sitta carolinensis } \\
\text { Sitta canadensis }\end{array}$ \\
\hline TROGLODYTIDAE & $\begin{array}{l}\text { Marsh Wren } \\
\text { Rock Wren } \\
\text { House Wren }\end{array}$ & $\begin{array}{l}\text { Cistothorus palustris } \\
\text { Salpinctes obsoletus } \\
\text { Troglodytes aedon }\end{array}$ \\
\hline $\begin{array}{l}\text { MUSCICAPIDAE } \\
\text { Sylviinae } \\
\text { Turdinae }\end{array}$ & $\begin{array}{l}\text { Golden-crowned Kinglet } \\
\text { Ruby-crowned Kinglet } \\
\text { Mountain Bluebird } \\
\text { Townsend's Solitaire } \\
\text { Veery } \\
\text { Hermit Thrush } \\
\text { Swainson's Thrush } \\
\text { American Robin }\end{array}$ & $\begin{array}{l}\text { Regulus satrapa } \\
\text { Regulus calendula } \\
\text { Sialia currucoides } \\
\text { Myadestes townsendi } \\
\text { Catharus fuscescens } \\
\text { Catharus guttatus } \\
\text { Catharus ustulatus } \\
\text { Turdus migratorius }\end{array}$ \\
\hline MIMIDAE & $\begin{array}{l}\text { Gray Catbird } \\
\text { Sage Thrasher }\end{array}$ & $\begin{array}{l}\text { Dumatella carolinensis } \\
\text { Oreoscoptes montanus }\end{array}$ \\
\hline MOTACILLIDAE & Water Pipit & Anthus spinoletta \\
\hline CINCLIDAE & American Dipper & Cinclus mexicanus \\
\hline
\end{tabular}




\begin{tabular}{|c|c|}
\hline BOMBYCILLIDAE & Cedar Waxwing \\
\hline STURNIDAE & European Starling \\
\hline VIREONIDAE & $\begin{array}{l}\text { Solitary Vireo } \\
\text { Warbling Vireo }\end{array}$ \\
\hline PASSERIDAE & House sparrow \\
\hline $\begin{array}{l}\text { EMBERIZIDAE } \\
\text { Parulinae }\end{array}$ & $\begin{array}{l}\text { Orange-crowned Warbler } \\
\text { Yellow-rumped Warbler } \\
\text { Townsend's warbler } \\
\text { Yellow Warbler } \\
\text { MacGillivray's Warbler } \\
\text { Wilson's Warbler } \\
\text { Northern Waterthrush } \\
\text { Common Yellowthroat } \\
\text { American Redstart }\end{array}$ \\
\hline Emberizinae & $\begin{array}{l}\text { Black-headed Grosbeak } \\
\text { Lazuli Bunting } \\
\text { Spotted towhee } \\
\text { Green-tailed Towhee } \\
\text { Vesper Sparrow } \\
\text { Savannah Sparrow } \\
\text { Song Sparrow } \\
\text { Lincoln's Sparrow } \\
\text { Fox Sparrow } \\
\text { Lark Sparrow } \\
\text { Chipping Sparrow } \\
\text { Clay-colored Sparrow } \\
\text { Brewer's Sparrow } \\
\text { Dark-eyed Junco } \\
\text { White-crowned Sparrow }\end{array}$ \\
\hline ICTERIDAE & $\begin{array}{l}\text { Bobolink } \\
\text { Western Meadowlark } \\
\text { Yellow-headed Blackbird } \\
\text { Red-winged Blackbird } \\
\text { Brewer' Blackbird } \\
\text { Brown-headed Cowbird } \\
\text { Common Grackle } \\
\text { Bullock's Oriole }\end{array}$ \\
\hline THRAUPIDAE & Western Tanager \\
\hline
\end{tabular}

Bombycilla cedrorum

Sturnus vulgaris

Vireo solitarius

Vireo gilvus

Passer domesticus

Vermivora celata

Dendroica coronata

Dendroica townsendi

Dendroica petechia

Oporornis tolmiei

Wilsonia pusilla

Seiurus novaboracensis

Geothlypis trichas

Setophaga ruticilla

Pheuticus melanocephalus

Passerina amoena

Pipilo maculatus

Pipilo chlorurus

Pooecetes gramineus

Passerculus sandwichensis

Melospiza melodia

Melospiza lincolnii

Passerella iliacus

Chondestes grammacus

Spizella paserina

Spizella pallida

Spizella breweri

Junco hyemalis

Zonotrichia leucophrys

Dolichonyx oryzivorus

Sturnella neglecta

Xanthocephalus xanthocephalus

Agelaius phoeniceus

Euphagus cyanocephalus

Molothru ater

Quiscalus quiscula

Icterus bullockii

Piranga ludoviciana 


\begin{tabular}{|l|l} 
FRINGILLIDAE & $\begin{array}{l}\text { Pine siskin } \\
\text { American Goldfinch } \\
\text { Red Crossbill } \\
\text { White-winged crossbill } \\
\text { Pine Grosbeak } \\
\text { Rosy Finch } \\
\text { Cassin's Finch } \\
\text { House Finch } \\
\text { Evening Grosbeak }\end{array}$ \\
& To Date: 153 species
\end{tabular}

Carduelis pinus

Carduelis tristis

Loxia curvirostra

Loxia leucoptera

Pinicola enucleator

Leucosticte arctoa

Carpodacus cassinii

Carpodacus mexicanus

Coccothraustes verpertinus 